\title{
Use of CytoSorb in the emergency department-high dependency unit: A case report and a mini review
}

\author{
Emanuela Biscardi, ${ }^{1,2}$ Giuseppe Carpinteri, ${ }^{1}$ Pietro Castellino, ${ }^{3}$ Lorenzo Malatino ${ }^{2}$ \\ ${ }^{1}$ Emergency Department, Azienda Ospedaliero Universitaria Policlinico G. Rodolico - San Marco, Catania; \\ ${ }^{2}$ Department of Clinical and Experimental Medicine, Postgraduate School of Emergency Medicine, \\ University of Catania, Cannizzaro Hospital, Catania; ${ }^{3}$ Department of Clinical and Experimental Medicine, \\ Azienda Ospedaliero Universitaria Policlinico G. Rodolico - San Marco, Catania, Italy
}

\begin{abstract}
Circulating inflammatory mediators and cytokines play a pivotal role in the progression of sepsis, leading in turn to septic shock, organ failure and resistance to standard therapy. Blood purification therapies may be adjuvant treatment for severe sepsis, but results have been shown to be so far controversial. Recently, CytoSorb has achieved promising outcomes on reduction of cytokine blood levels, improvement of clinical parameters and
\end{abstract}

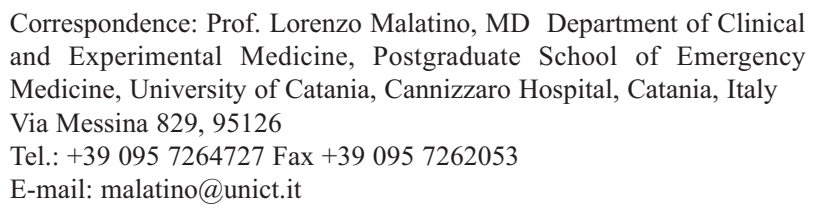

Key words: CytoSorb; Emergency department-high dependency unit; septic shock; cytokines; rescue therapy.

Acknowledgements: The authors thank Dr. Salvatrice Platania, MD, Nephrologist, for her help in the use of CytoSorb.

Contributions: EB acquired the data and drafted the initial manuscript; GC planned the project and provided some important bibliographic references; PC supervised data collection and data analysis and LM critically revised the manuscript and bibliography. All authors approved the final manuscript as submitted and are accountable for all aspects of the manuscript as submitted.

Conflict of interest: The authors declare no conflict of interest.

Availability of data and materials: All data underlying the findings are fully available.

Ethics approval and consent to participate: No ethical committee approval was required for this case report by the Department, because this article does not contain any studies with human participants or animals. Informed consent was obtained from the patient included in this study.

Consent for publication: The patient gave her written consent to use her personal data for the publication of this case report and any accompanying images.

Received for publication: 22 April 2021.

Revision received: 1 September 2021.

Accepted for publication: 23 September 2021.

This work is licensed under a Creative Commons Attribution 4.0 License (by-nc 4.0)

( Copyright: the Author(s), 2021

Licensee PAGEPress, Italy

Emergency Care Journal 2021; 17:9825

doi:10.4081/ecj.2021.9825 mortality in sepsis, as well as in other acute conditions. It is mostly used in Intensive Care Unit (ICU), in isolated hemoperfusion, or inserted in other circuits in addition to Continuous Renal Replacement Therapy (CRRT), heart-lung machines and extracorporeal membrane oxygenation. We report a case of septic shock occurred in our Emergency Department-High Dependency Unit (ED-HDU), resistant to standard therapy and improved after CytoSorb, so avoiding ICU hospitalization.

\section{Highlights}

CytoSorb is a rescue therapeutic option for septic shock. CytoSorb is a safe, simple, well-tolerated, no haemolytic device.

- CytoSorb reduces cytokine blood levels in septic shock, so leading to hemodynamic stabilization.

- Adequately staffed Emergency Departments-High Dependency Units are decisive to manage critical patients, possibly avoiding Intensive Care Unit (ICU) admission and sparing ICU's resources.

\section{Case Report}

A 34-years-old woman with lupus nephritis, on chronic therapy with immunosuppressants, was admitted to the Emergency Department (ED) with fever, cough and anuria. Her past medical history revealed previous hospitalizations for lupus nephritis exacerbations with neurological involvement. We started routine diagnostic work-up as follows: blood exams, blood cultures, 12-lead ECG, pulse oximetry, invasive arterial blood pressure measurement, hourly monitoring of diuresis, temperature measurement and gas records. Gas exams showed combined metabolic acidosis and respiratory alkalosis, as expected. Blood exams showed anemia, AKI and high inflammatory indexes with a Sequential Organ Failure Assessment (SOFA) score 8. Severe hypotension, anuria and SOFA score made likely a septic status. An initial ultrasound assessment revealed bilateral pleural effusion, lung A lines predominance and Inferior Vena Cava (IVC) of $2 \mathrm{~cm}$, with respiratory collapsibility of $40 \%$. No significant echocardiographic alterations were found. Hence, immunosuppressants were stopped and therapy with fluids and broad-spectrum antibiotic was started. A chest CT scan was performed showing bilateral pneumonia and blood transfusion was also performed. Oxygen with High Flow Nasal Cannula (HFNC) was also supplied. Due to the persistence of hemodynamic instability, norepinephrine was added to the infusion therapy and diuretic was started. In order to give continuous close monitoring, the patient was moved from ED to our ED-HDU, a 
sub-intensive care unit located between Emergency Room (ER) and ICU, with availability of advanced monitoring, non-invasive ventilation and possibility to give vasoactive drugs. After 24 hours, blood cultures were positive for S. Pneumoniae. Anuria, hypotension and high inflammation indexes persisted. We potentiated vasopressor support and we considered starting Continuous VenoVenous Hemodiafiltration (CVVHDF) in order to maintain hydroelectrolytic homeostasis, prevent further renal insult and allow antibiotic therapy without limitations or complications. Taking into account the presence of dialysis devices and nephrology consultants in our ED-HDU, informed consent was obtained from the patient and a femoral Central Venous Catheter (CVC) was implanted. CVVHDF was performed for 48 hours as standalone therapy, using a renal replacement device (Prismaflex- Baxter), with heparin anticoagulation and a blood flow rate of $200 \mathrm{~mL} / \mathrm{h}$. After 48 hours from CVVHDF, assuming that the persistence of AKI with anuria and elevated inflammatory markers, above all Procalcitonin (PCT), were possibly related to cytokine storm, CytoSorb cartridge was placed downstream of the blood pump circuit, with a blood flow rate of $200 \mathrm{~mL} / \mathrm{h}$. Hence, CytoSorb was not started from the beginning, but only when the balance of clinical status was considered to be tipped towards a cytokine storm. This treatment was conducted intermittently for two consecutive sessions, each lasting 10 hours. Fluid load was closely monitored daily through bedside echo. Between CytoSorb sessions, ultrafiltration in CVVHDF modality was also performed as needed to control fluid homeostasis. The patient tolerated very well the procedure with no sideeffects and was transferred to the Unit of Internal Medicine, so avoiding ICU admission. The effect of CVVHDF without CytoSorb consisted of unstable improvement of blood pressure, consequently still requiring norepinephrine infusion, associated with a slight reduction of inflammatory indexes and SOFA score. By contrast, CVVDHF with CytoSorb allowed hemodynamic stabilization with no further norepinephrine demand, associated with CRP, PCT and SOFA score reduction. During the renal replacement treatment, norepinephrine infusion was gradually stopped and the patient preserved a good oxygen saturation with High Flow Nasal Cannula (HFNC), so that mechanical ventilation was not necessary. CytoSorb allowed a reduction of the vasopressor dose and hemodynamic stabilization, with gradual recovery of spontaneous diuresis. The patient was monitored at baseline, at 24, 48 and 72 hours and blood samples were drawn to measure a number of parameters (Table 1).

\section{Discussion}

AKI is one of the most important septic complication representing an independent risk factor for mortality. During septic condition, elevated and imbalanced pro- and anti-inflammatory mediators, coupled with severe endothelial dysfunction and a perturbed coagulation cascade, works synergistically to induce chemicallyand biologically-mediated kidney injury. Renal Replacement Therapies (RRTs) have been tested to restore a balanced immune response by clearing/deactivating inflammatory mediators ${ }^{1}$ but they have not to date been successful and are not widely utilized in practice, depending on resources and local expertise. ${ }^{2}$ Table 2 shows the core differences of the several different purification systems used in sepsis. ${ }^{2-6}$ The indications for the use of RRT in sepsis-associated AKI are consistent with those for other causes of AKI but, given the potential confounding effects of sepsis on conventional markers, some investigators have suggested prolonged oliguria as sufficient indication to start RRT. The use of continuous therapies in sepsis-associated AKI are still preferred managing to improve hemodynamic stability, temperature regulation and continuous fluid management. Of note, also the polymer-based sorbents have shown to be effective in restoring immune homeostasis in septic patients. ${ }^{3}$ CytoSorb is one of the several sorbents developed and approved in Europe since 2011 for inflammatory mediators removal. It was used for the first time in 2014 in combination with CRRT, to treat septic shock secondary to beta-hemolytic Streptococcus-induced necrotizing fasciitis. ${ }^{7}$ Later on, some case series have been reported in the Literature, but its use has not been so far recognized as a mandatory approach to septic shock. Technically simple and biocompatible, CytoSorb can be entered in most circuits and needs little adjustment without adverse effects, avoiding hemolysis. CytoSorb has a surface of about $45,000 \mathrm{~m}^{2}$, wider than that of the others filters, allowing a rapid adsorption of hydrophobic molecules with a molecular weight between 5 and 55 $\mathrm{kDa}$. Hence, CytoSorb is selective for cytokines, leading to a decrease of key cytokines such as IL- $1 \beta$, TNF- $\alpha$, IL- 6 and IL-10 both in ICU patients 8,9 and laboratory septic rats. ${ }^{10}$ Endotoxins coming from Gram negative bacteria have higher molecular weight $(100 \mathrm{kDa})$, so they are not removed by CytoSorb. The adsorption is concentration-dependent: the higher are the levels of substances in the blood, the faster they are cleared. Literature provides evidence about reduction of high levels of cytokines, mostly IL-6 ${ }^{2,9,11,12}$ but results are controversial with no decrease in IL-6 plasma levels and no association with mortality, ${ }^{13}$ probably due to influence of treatment duration, cytokines' continuous production and their shift from interstitium into blood..$^{9,14}$ Moreover, CytoSorb can reduce levels of PCT and $\mathrm{CRP}^{12,15}$ which have received, above all, a large consensus as being considered sepsis biomarkers. ${ }^{16}$ PCT has been considered a biomarker of "cytokine storm": the higher is the value of PCT, the higher are cytokines levels. Despite its reduction determines improvement in clinical parameters, ${ }^{15}$ it is difficult to establish a PCT cut-off. Nevertheless, PCT could indicate when to start sorbent therapy, but not to follow the response to therapy itself. ${ }^{14}$ Recent studies have shown that CytoSorb can promote hemodynamic stabilization with a reduction of catecholamines demand, increasing short-term survival and reducing mortality predicted by scores. ${ }^{8,17}$ Of note, in patients with refractory septic shock, no responder to fluid challenge, vasopressors are important to reach a Mean Arterial Pressure (MAP) $\geq 65 \mathrm{mmHg}$ and guarantee organ perfusion. However, vasopressor load and prolonged titrated therapy may positively correlate with organ ischemia, tachyarrhythmia, metabolic acidosis and renal failure. ${ }^{18}$ CytoSorb influences vasopressors need through the control of the exaggerated immune response and the reduction of inflammatory mediators, which typically lead to hemodynamic stability. In turn, patients with high severity of illness and refractory shock are more likely to benefit of sorbent therapy. Improved hemodynamic associated with a reduction in catecholamine requirements has been acknowledged as a relevant endpoint of sorbent therapy. ${ }^{14,17,19}$ These results derive from combination between CytoSorb and CRRT, but also from CytoSorb as standalone therapy. ${ }^{15}$ Also, it is well recognized that the serum lactate level is helpful in identifying occult shock and in monitoring response to therapy. ${ }^{8,11,12,20}$ In turn, recent studies have shown that, in patients treated with CytoSorb, a baseline lactate level of 6-7.5 and above, together with low thrombocytes count, are associated with higher mortality. ${ }^{17,19}$ It is necessary keeping in mind that CytoSorb may interfere with drug kinetics (especially that of lipophilic antibiotics), with unwanted effects (e.g., sub-therapeutic antibiotics concentration that may dampen their efficacy). To date, there is scanty information surrounding the 
Table 1. Changes in laboratory and vital parameters.

\begin{tabular}{|c|c|c|c|c|c|c|c|}
\hline \multirow[t]{2}{*}{ Parameters } & \multirow[t]{2}{*}{$\mathbf{O} / \mathbf{A}$} & \multirow[t]{2}{*}{ ST } & \multirow{2}{*}{$\begin{array}{l}\text { CVVHDF } \\
+ \text { ST }\end{array}$} & \multicolumn{2}{|c|}{$\begin{array}{l}\text { CytoSorb } \\
\text { therapy }\end{array}$} & \multicolumn{2}{|c|}{ Stop CytoSorb } \\
\hline & & & & $\mathrm{T}_{0}$ & $\mathrm{~T}_{24}$ & $\mathrm{~T}_{48}$ & $\mathrm{~T}_{72}$ \\
\hline $\mathrm{BP}(\mathrm{mmHg})$ & $70 / 35$ & $70 / 40$ & $96 / 65$ & $90 / 60$ & $100 / 70$ & $125 / 70$ & $145 / 95$ \\
\hline PCT (ng/mL) & 25.03 & 98.17 & 33.91 & 23.61 & 20.61 & 7.62 & 4.07 \\
\hline CRP (mg/L)(0.0-1.0) & 10.574 & 22.285 & 41.187 & 39.033 & 19.582 & 10.532 & 5.1 \\
\hline $\mathrm{WBC}\left(10^{3} / \mu \mathrm{L}\right)(4.80-10.80)$ & 4.18 & 4.9 & 9.32 & 13.4 & 17.82 & 14.82 & 15.60 \\
\hline$-\mathrm{Ne}(\%)(40-74)$ & 88.1 & 88.8 & 93.7 & 95.2 & 91.2 & 86.4 & 86.7 \\
\hline$-\mathrm{Li}(\%)(19-48)$ & 8.4 & 9.8 & 5.0 & 2.1 & 4.0 & 5.7 & 4.7 \\
\hline -Mo (\%) (3.4-9) & 3.0 & 1.1 & 0.8 & 2.6 & 4.7 & 7.8 & 8.4 \\
\hline -Eo (\%) (0.5-7) & 0.3 & 0.1 & 0.3 & 0 & 0 & 0 & 0.1 \\
\hline$-\mathrm{Ba}(\%)(0-1.5)$ & 0.2 & 0.2 & 0.2 & 0.1 & 0.1 & 0.1 & 0.1 \\
\hline eGFR $\left(\mathrm{mL} / \mathrm{min} / \mathrm{m}^{2}\right)$ & 28 & 19 & 45 & 70 & 43 & 54 & 58 \\
\hline Serum Urea $(\mathrm{mg} / \mathrm{dL})(17-43)$ & 110 & 126 & 63 & - & 76 & 93 & 134 \\
\hline $\begin{array}{l}\mathrm{SCr}(\mathrm{mg} / \mathrm{dL}) \\
(0.60-1.10)\end{array}$ & 2.22 & 3.08 & 1.52 & 1.06 & 1.59 & 1.31 & 1.24 \\
\hline PT-INR (0.8-1.2) & 1.04 & 1.25 & 1.11 & 0.93 & 0.94 & 0.88 & 0.89 \\
\hline PLT $\left(10^{3} / \mu \mathrm{L}\right)$ & 222 & 204 & 243 & 205 & 178 & 142 & 136 \\
\hline APTT (sec)/Ratio (40/1.33) & $33 / 1.09$ & $35 / 1.18$ & $47 / 1.55$ & $46 / 1.53$ & $72 / 2.39$ & $29 / 0.97$ & $40 / 1.33$ \\
\hline $\begin{array}{l}\mathrm{Lac}(\mathrm{mmol} / \mathrm{L}) \\
(1.0-1.8)\end{array}$ & 2.8 & 2.2 & 1.6 & 1.5 & 1.3 & - & 1.3 \\
\hline SOFA score $(<2)$ & 8 & 7 & 7 & 5 & 3 & - & 2 \\
\hline
\end{tabular}

O/A: On Arrival; ST: Standard Therapy; CWHDF+ ST: Continuous Veno-venous Hemodiafiltration + Standard Therapy; BP: Blood Pressure; PCT: Procalcitonin; CRP: C-Reactive Protein; WBC: white blood cells; Ne: Neutrophils; Ly: Lymphocytes; Mo: Monocytes; Eo: Eosinophils; Ba: Basophils; eGFR: Estimated Glomerular Filtration Rate; SCr: Serum Creatinine; PT-INR: Prothrombin Time International Normalized Ratio; PLT: Platelets; APTT: Activated Partial Thromboplastin Time; Lac: Lactate; SOFA: Sequential Organ Failure Assessment. 
Table 2. Core differences of several blood purification therapies used in sepsis.

\begin{tabular}{|c|c|c|}
\hline & IRRT & $\begin{array}{l}\text { CRRT: CVVH, CVVHD, CVVHDF, SCUF, HP, hybrid } \\
\text { CPFA, PEX }\end{array}$ \\
\hline Mechanism & $\begin{array}{l}\text { Diffusive } \\
\text { transport (HD) } \\
\text { or convection } \\
\text { (HF, HVHF) } \\
\text { or both (HDF) } \\
\text { Presence of a } \\
\text { dialysate }\end{array}$ & $\begin{array}{l}\text { Mainly Convective /Ultrafiltration transport } \\
\text { (except for CVVHD diffusion and CVVHDF } \\
\text { combination of convection and diffusion). Presence } \\
\text { of sterile replacement solution (not for CVVHD } \\
\text { and SCUF). } \\
\text { Adsorption of blood (HP) or combination of } \\
\text { convective with plasma filtration and adsorption } \\
\text { (CPFA) } \\
\text { Filtration with reinfusion of albumin or fresh } \\
\text { frozen plasma (PEX) }\end{array}$ \\
\hline Effect & $\begin{array}{l}\text { Clearance for } \\
\text { small } \\
\text { molecular } \\
\text { weight solutes } \\
\text { and fluids }\end{array}$ & $\begin{array}{l}\text { Clearance for small and middle molecular weight solutes } \\
\text { and fluids (CVVHDF). Only fluids for SCUF. } \\
\text { Cytokines or poisons or other toxins removal depending on } \\
\text { the sorbent (HP): CytoSorb: pro and anti-inflammatory } \\
\text { cytokines removal except of endotoxins; Polimixine B: } \\
\text { endotoxins removal; Alteco: LPS adsorber; oXiris: } \\
\text { endotoxins removal } \\
\text { Pathological substances (cytokines, autoantibodies, toxins, } \\
\text { abnormal proteins) removal (PEX) }\end{array}$ \\
\hline Fluid removal & Rapid & $\begin{array}{l}\text { - CVVHDF: slow } \\
\text { - Sorbents: not rely on fluids removal }\end{array}$ \\
\hline
\end{tabular}


Table 2. Core differences of several blood purification therapies used in sepsis.

\begin{tabular}{|c|c|c|}
\hline & & - PEX: slow large fraction of plasma filtration \\
\hline Flow rate & $\begin{array}{l}\text { High (up to } \\
500 \mathrm{~mL} / \mathrm{min} \text { ) }\end{array}$ & $\begin{array}{l}\text { Low and constant ( } 50-200 \mathrm{~mL} / \mathrm{min}) \text {. High intensity } \\
\text { dialysis }(40 \mathrm{mg} / \mathrm{kg} / \mathrm{h}) \text { does not change the mortality } \\
\text { compared to a less intensive treatment }(20 \mathrm{mg} / \mathrm{kg} / \mathrm{h})\end{array}$ \\
\hline Hypotension & High risk & Present but lower risk \\
\hline Complexity & Low & High \\
\hline $\begin{array}{l}\text { Systemic } \\
\text { Anticoagulation }\end{array}$ & Not necessary & Present (heparin or citrate) \\
\hline Disadvantages & $\begin{array}{l}\text { Frequent } \\
\text { hypotension, } \\
\text { arrythmia, } \\
\text { hypertension, } \\
\text { hyperkalemia }\end{array}$ & $\begin{array}{l}\text { CVVHDF: filter clotting, air embolism, } \\
\text { hypothermia, bleeding, drug levels alteration, } \\
\text { prolonged immobilization, hypotension (less } \\
\text { frequent than IRRT) } \\
\text { - Sorbents: drug kinetic interference, transient } \\
\text { thrombocytopenia and leucopenia } \\
\text { PEX: vascular access problems, paresthesia, } \\
\text { anaphylactoid reaction to replacement fluid, } \\
\text { hypotension, nausea, vomiting, thrombocytopenia }\end{array}$ \\
\hline Costs & Low/moderate & High \\
\hline
\end{tabular}

IRRT: Intermittent Renal Replacement Therapy; HD: Hemodialysis; HF: Hemofiltration; HDF: Hemodiafiltration; HVHF: High volume hemofiltration; CRRT: Continuous Renal Replacement Therapy; CVH: Continuous Veno-Venous Hemodialysis; CWHD: Continuous Veno-Venous Hemofiltration; CVHDF: Continuous Veno-Venous Hemodiafiltration; SCUF: Slow Continuous Ultrafiltration; HP: Hemoperfusion; CPFA: Coupled plasma filtration absorption; PEX: Plasmaexchange 
use of hemadsorption therapies in septic shock, limited to animal studies, case reports, and retrospective observational studies. These data cannot provide clear recommendations for the use of CytoSorb. Further studies should be useful to better define indication to use, timing and therapeutic efficacy of sorbent agents. Actually, sorbent therapy should not be based only on the presence of high biomarkers level, but also on the monitoring of severity of illness (through APACHE and SOFA scores), norepinephrine need, mortality, length of stay and mechanical ventilation. ${ }^{9}, 14$ In this scenario, it is important to develop a unique assessment to objectively evaluate the most appropriate patient's care in the emergency setting and the impact of clinical interventions. ${ }^{21}$ In the context of ED-HDU, SOFA score has shown promising results in terms of prognostic value, response to therapeutic intervention and discrimination ability for HDU mortality and indication to ICU admission. ${ }^{22,23}$ Moreover, additional modified indexes such as qSOFA (Quick Sequential Organ Failure Assessment) and LqSOFA (blood lactate levels + qSOFA) resulted to be useful in predicting, with high sensitivity and specificity, mortality in septic patients, when calculated on admission to ED. ${ }^{24}$

\section{Conclusions}

To the best of our knowledge, there is no previous experience in the use of CytoSorb in an ED-HDU and this is the novelty of the present clinical case. We believe that the presence of ED-HDU with dialysis device has been a winner strategy to manage our patient in the context of sub-intensive care, avoiding ICU admission. In life-threatening conditions like septic shock, RRT is usually under the domain of ICUs. ${ }^{8,11}$ Hence, the management of critically ill patients is considered a synonymous of ICU's expertise, but relevant therapy in ED may improve mortality scores and physiologic assessment prior to ICU admission. Therefore, length of stay in ED is often prolonged because of lack of ICU beds, ED overcrowding, ageing population, complexity of some chronic conditions leading to time-dependent complications and increase of mortality scores. Even better, the efficacy of ED intervention may result in avoiding ICU admission and sparing ICU's resources. Hence, ED must be staffed and organized adequately to manage stabilization procedures. ${ }^{25,26}$ This case report strengthens the effectiveness of approaching patients with septic shock in the emergency department not only as first aid but as conclusive therapeutic strategy, whenever possible. We believe that the quality of assistance provided during acute management significantly inhibits the progression of organ failure and death, irrespective of patients' location. Waiting for ICU admission without adequate resuscitation strategy may cause dramatic complications leading to death. On the other side, ICU mortality and ICU related long-term complications are documented, mostly due to infections, mechanical ventilation or premature discharge due to beds pressure. ${ }^{27}$ Lastly, this manuscript may encourage the use of CytoSorb, when indicated, during the acute phase of medical care, because it is a safe, promising and well tolerated rescue therapeutic option in septic shock.

\section{References}

1. Ronco C, Kellum JA, Bellomo R, House AA. Potential interventions in sepsis-related acute kidney injury. Clin J Am Soc Nephrol 2008;3:531-44.
2. Ankawi G, Neri M, Zhang J, et al. Extracorporeal techniques for the treatment of critically ill patients with sepsis beyond conventional blood purification therapy: the promises and the pitfalls. Crit Care 2018;22:262.

3. Forni LG, Ricci Z, Ronco C. Extracorporeal renal replacement therapies in the treatment of sepsis: where are we? Semin Nephrol 2015;35:55-63.

4. Girardot T, Schneider A, Rimmelé T. Blood purification techniques for sepsis and septic AKI. Semin Nephrol 2019;39:50514.

5. Dutto L, Melchio R. Reviews from literature and web. Emerg Care J 2009;5:42.

6. RENAL Replacement Therapy Study Investigators, Bellomo $\mathrm{R}$, Cass A, et al. Intensity of Continuous Renal-Replacement Therapy in Critically Ill Patients. N Engl J Med 2009;361:1627-38.

7. Hetz H, Berger R, Recknagel P, Steltzer H. Septic shock secondary to $\beta$-hemolytic streptococcus-induced necrotizing fasciitis treated with a novel cytokine adsorption therapy. Int $\mathrm{J}$ Artif Organs 2014;37:422-6.

8. Kogelmann K, Jarczak D, Scheller M, Drüner M. Hemoadsorption by CytoSorb in septic patients: a case series. Crit Care 2017;21:74.

9. Morris C, Gray L, Giovannelli M. Early report: The use of Cytosorb $^{\mathrm{TM}}$ haemabsorption column as an adjunct in managing severe sepsis: initial experiences, review and recommendations. J Intensive Care Soc 2015;16:257-64.

10. Kellum JA, Song M, Venkataraman R. Hemoadsorption removes tumor necrosis factor, interleukin-6, and interleukin10 , reduces nuclear factor- $\mathrm{kB}$ DNA binding, and improves short-term survival in lethal endotoxemia*. Crit Care Med 2004;32:801-5.

11. Friesecke S, Träger K, Schittek GA, et al. International registry on the use of the CytoSorb ${ }^{\circledR}$ adsorber in ICU patients. Medizinische Klin - Intensivmed und Notfallmedizin 2019;114:699-707.

12. Mehta Y, Mehta C, Kumar A, et al. Experience with hemoadsorption (CytoSorb $\left.{ }^{\circledR}\right)$ ) in the management of septic shock patients. World J Crit Care Med 2020;9:1-12.

13. Schädler D, Pausch C, Heise D, et al. The effect of a novel extracorporeal cytokine hemoadsorption device on IL-6 elimination in septic patients: A randomized controlled trial. Eller K, editor. PLoS One 2017;12:e0187015.

14. Honore PM, Hoste E, Molnár Z, et al. Cytokine removal in human septic shock: Where are we and where are we going? Ann Intensive Care 2019;9:56.

15. Hawchar F, László I, Öveges N, et al. Extracorporeal cytokine adsorption in septic shock: A proof of concept randomized, controlled pilot study. J Crit Care 2019;49:172-8.

16. Lippi G, Montagnana M, Balboni F, et al. Academy of Emergency Medicine and Care-Society of Clinical Biochemistry and Clinical Molecular Biology consensus recommendations for clinical use of sepsis biomarkers in the emergency department. Emerg Care J 2017;13:6877.

17. Brouwer WP, Duran S, Kuijper M, Ince C. Hemoadsorption with CytoSorb shows a decreased observed versus expected 28-day all-cause mortality in ICU patients with septic shock: a propensity-score-weighted retrospective study. Crit Care 2019;23:317.

18. Russell JA. Vasopressor therapy in critically ill patients with shock. Intensive Care Med 2019;45:1503-17.

19. Rugg C, Klose R, Hornung R, et al. Hemoadsorption with CytoSorb in septic shock reduces catecholamine requirements 
and in-hospital mortality: a single-center retrospective 'genetic' matched analysis. Biomedicines 2020;8:539.

20. Coen D. Hyperlactatemia and hypotension: looking at septic shock from different perspectives. Emerg Care J 2013;9:9.

21. Nguyen HB, Rivers EP, Havstad S, et al. Critical care in the emergency department a physiologic assessment and outcome evaluation. Acad Emerg Med 2000;7:1354-61.

22. Innocenti F, Tozzi C, Donnini C, et al. SOFA score in septic patients: incremental prognostic value over age, comorbidities, and parameters of sepsis severity. Intern Emerg Med 2017;13:405-12.

23. Innocenti F, Caldi F, Tassinari I, et al. SOFA Score prognostic performance among patients admitted to High-Dependency Units. Minerva Anestesiol 2019;85:1080-8.
24. Velissaris D, Karamouzos V, Pantzaris ND, et al. qSOFA score and LqSOFA score as predictors of outcome in an elderly population with chest infection treated in the Emergency Department. A case series. Emerg Care J 2019;15:8235.

25. Rivers E, Nguyen B, Havstad S, et al. Early goal-directed therapy in the treatment of severe sepsis and septic shock. N Engl J Med 2001;345:1368-77.

26. O'Connor G, Geary U, Moriarty J. Critical care in the emergency department. Eur J Emerg Med 2009;16:296-300.

27. Capuzzo M, Volta CA, Tassinati T, et al. Hospital mortality of adults admitted to Intensive Care Units in hospitals with and without Intermediate Care Units: a multicentre European cohort study. Crit Care 2014;18:551. 